\title{
ON 3-HYPERGRAPHS WITH FORBIDDEN 4-VERTEX CONFIGURATIONS*
}

\author{
ALEXANDER A. RAZBOROV ${ }^{\dagger}$
}

\begin{abstract}
Every 3-graph in which no four vertices are independent and no four vertices span precisely three edges must have edge density $\geq 4 / 9(1-o(1))$. This bound is tight. The proof is a rather elaborate application of Cauchy-Schwarz-type arguments presented in the framework of flag algebras. We include further demonstrations of this method by re-proving a few known tight results about hypergraph Turán densities and significantly improving numerical bounds for several problems for which the exact value is not known yet.
\end{abstract}

Key words. extremal combinatorics, Turán problem, flag algebras

AMS subject classifications. 05C65, 05D99, 13P25, 90C22

DOI. $10.1137 / 090747476$

1. Introduction. In the classical paper [14], Mantel determined the minimal number of edges a graph $G$ must have so that every three vertices span at least one edge. This minimum is attained if we take an almost balanced function $\chi: V(G) \longrightarrow$ $\{0,1\}$ and let $E(G)$ consist of all $\chi$-monochromatic edges. In the paper [25] (that essentially started off the field of extremal combinatorics), Turán generalized Mantel's result to independent sets of arbitrary size. He also asked if similar generalizations can be obtained for hypergraphs, and these questions became notoriously known ever since as one of the most difficult open problems in discrete mathematics. But they also became pivotal for its development.

To be more specific (many more details can be found in the survey [24]), let $I_{\ell}^{r}$ be the empty $r$-uniform hypergraph ( $r$-graph in what follows) on $\ell$ vertices. For $r$-graphs $H_{1}, \ldots, H_{h}$, we let

$$
\pi_{\min }\left(H_{1}, \ldots, H_{h}\right) \stackrel{\text { def }}{=} \lim _{n \rightarrow \infty} \frac{\operatorname{ex}_{\min }\left(n ; H_{1}, \ldots, H_{h}\right)}{\left(\begin{array}{l}
n \\
r
\end{array}\right)},
$$

where $\operatorname{ex}_{\min }\left(n ; H_{1}, \ldots, H_{h}\right)$ is the minimal possible number of edges in an $n$-vertex $r$-graph not containing any of $H_{1}, \ldots, H_{h}$ as an induced subgraph.

Remark 1. The standard Turán density $\pi\left(H_{1}, \ldots, H_{h}\right)$ is defined analogously, with the differences that we are looking for the maximal number of edges, and that we forbid all copies of $H_{1}, \ldots, H_{h}$, not necessarily induced. In general, we only have the relation

$$
\pi\left(H_{1}, \ldots, H_{h}\right) \leq 1-\pi_{\min }\left(\bar{H}_{1}, \ldots, \bar{H}_{h}\right),
$$

where $\bar{H}_{i}$ is the $r$-graph complementary to $H_{i}$. But when the family $\left\{H_{1}, \ldots, H_{h}\right\}$ is closed under edge deletion, the difference between induced and noninduced becomes inessential, and, in particular, we have

$$
\pi\left(K_{\ell}^{r}\right)=1-\pi_{\min }\left(I_{\ell}^{r}\right)
$$

\footnotetext{
${ }^{*}$ Received by the editors January 22, 2009; accepted for publication (in revised form) June 20, 2010; published electronically August 12, 2010.

http://www.siam.org/journals/sidma/24-3/74747.html

$\dagger$ Department of Computer Sciences, University of Chicago, Chicago, IL 60637 (razborov@cs. uchicago.edu). Part of this work was done while the author was with Steklov Mathematical Institute, supported by the Russian Foundation for Basic Research, and with Toyota Technological Institute at Chicago.
} 
where $K_{\ell}^{r}$ is the complete $r$-graph on $\ell$ vertices. In any case, we prefer the treatment in complementary terms as it is more consistent with some of the relevant literature and makes constructions look slightly neater.

We still do not know $\pi_{\min }\left(I_{\ell}^{r}\right)$ (not even to mention the exact value of $\operatorname{ex}_{\min }\left(n ; I_{\ell}^{r}\right)$ ) for any pair $\ell>r \geq 3$, although plausible conjectures do exist [24]. The simplest unresolved case that has also received the most attention is $r=3, \ell=4$. Turán himself proved that $\pi_{\min }\left(I_{4}^{3}\right) \leq 4 / 9$ and conjectured that this is actually the right value. His construction goes as follows: fix an almost balanced function $\chi: V(H) \longrightarrow \mathbb{Z}_{3}$, and let $E(H)$ consist of all those triples $e \subseteq V(H)$ for which one of the following is true:

1. $e$ is $\chi$-monochromatic;

2. there exists $a \in \mathbb{Z}_{3}$ such that $\left.\chi\right|_{e}$ takes on the value $a$ two times, and the value $a+1$ one time.

Brown [4] found another example attaining the edge density 4/9, and Kostochka [12] significantly generalized both these constructions by presenting a "continuous" series of examples witnessing the inequality $\pi_{\min }\left(I_{4}^{3}\right) \leq 4 / 9$. These are all currently known "asympotically different" constructions achieving the edge density $4 / 9$ (or, in terms of flag algebras, all different homomorphisms $\phi \in \operatorname{Hom}^{+}\left(\mathcal{A}^{0}, \mathbb{R}\right)$ with $\phi(\rho)=$ $4 / 9$ ).

Fon-der-Flaass [8] offered a very instructive interpretation of Kostochka's examples. More precisely, he showed how to convert any simple digraph with no induced $\vec{C}_{4}$ into a 3 -graph with no induced $I_{4}^{3}$; Kostochka's examples can all be constructed in this way from rather simple digraphs. References [6], Giraud (unpublished), and [7] proved increasingly stronger lower bounds on $\pi_{\min }\left(I_{4}^{3}\right)$, with the current (to the best of our knowledge) record

$$
\pi_{\min }\left(I_{4}^{3}\right) \geq \frac{9-\sqrt{17}}{12} \geq 0.406407
$$

held by [7].

Let $G_{i}$ be the uniquely defined 3 -graph on 4 vertices with $i$ edges. An easy inspection of Turán's construction reveals that besides $I_{4}^{3}$ (which is $G_{0}$ ) it also misses $G_{3}$ as an induced subgraph. Therefore, that construction also proves $\pi_{\min }\left(I_{4}^{3}, G_{3}\right) \leq$ 4/9. The main result of this paper is that this bound is actually tight.

TheOREM 1. $\pi_{\min }\left(I_{4}^{3}, G_{3}\right)=4 / 9$. Or, in complementary terms, every 3-graph on $n$ vertices that does not contain complete subgraphs on 4-vertices, and in which no 4 vertices span exactly one edge, must have $\leq\left(\begin{array}{l}n \\ 3\end{array}\right)\left(\frac{5}{9}+o(1)\right)$ edges.

Our proof is the second application of the formalism of flag algebras developed in [22], but this time we exploit features of this framework quite different from those exploited in the first application [23]. In fact, we simply apply a Cauchy-Schwarz argument (aka semidefinite method) similar to those that were many times applied in this area in the past. However, the mathematical structure inherent in the theory of flag algebras allowed us to computerize the search for "right" relations using Maple and the CSDP package for semidefinite programming [3]. As the reader will hopefully be convinced below, this has endowed us with capabilities (in terms of the sheer amount of routine work that becomes feasible) which can hardly be attained with any non-systematic approach. And we would like to specifically acknowledge here the earlier paper by Bondy [2] devoted to similar goals in the context of a different problem.

Finally, we applied the same semidefinite program to Turán's original problem, and our numerical computations suggest the following improvement of (1):

$$
\pi_{\min }\left(I_{4}^{3}\right) \geq 0.438334 \text {. }
$$




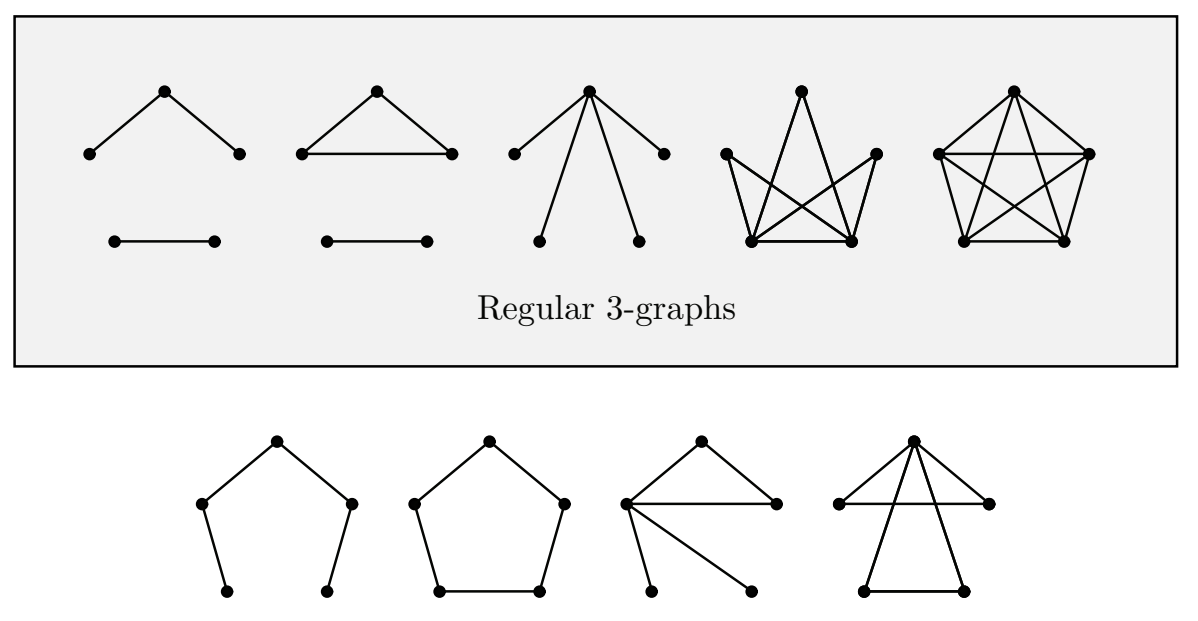

FIG. 1. Admissible 3-graphs on 5 vertices.

We, however, did not feel motivated enough (there are 964 nonisomorphic 3-graphs on 6 vertices without induced $I_{4}^{3}$ !) to try to convert this floating-point computation into a rigorous mathematical proof.

The rest of the paper is organized as follows. In section 2 we recall the small fragment of the theory of flag algebras that is needed for our purposes (mostly notational system; in this paper we do not need its more sophisticated parts). Our account is specialized to the concrete first-order theory (of 3-graphs without induced $G_{0}$ or $G_{3}$ ), and we give plenty of examples as we go along. Section 3 contains the proof of our main result. We deliberately present it first in the form of an ad hoc computation in flag algebras. For a (loose) description of the heuristical process and some mathematics behind these calculations that might be useful in other situations, the interested reader is referred to section 4 . We finish with a few concluding remarks in section 5 .

2. Flag algebras. We call a 3-graph admissible if it does not contain induced copies of $G_{0}$ or $G_{3}$ (recall that $G_{i}$ is the 3 -graph on 4 vertices with $i$ edges). Let $\mathcal{H}_{\ell}$ be the set of all admissible 3 -graphs on $\ell$ vertices considered up to an isomorphism.

$\mathcal{H}_{3}$ consists of two elements: an edge and the empty 3 -graph. We will denote the first of them (the edge) by $\rho$.

$$
\mathcal{H}_{4}=\left\{G_{1}, G_{2}, G_{4}\right\} \text {. }
$$

$\mathcal{H}_{5}$ has 9 elements. We depict them on Figure 1 using the following convenient notation from [7]: a 3 -graph on [5] $\stackrel{\text { def }}{=}\{1,2, \ldots, 5\}$ corresponds to the ordinary graph with the same vertex set [5] in which $e \in[5]^{2}$ is an edge if and only if $\{1,2, \ldots, 5\} \backslash e$ is a (hyper)edge of the original 3-graph. This creates one-to-one correspondence between $\mathcal{H}_{5}$ and the set of (ordinary) graphs on 5 vertices without vertices of degree 0 or 3 shown on Figure 1. The five 3 -graphs in the shaded area will be called regular; these are precisely those that actually appear in Turán's construction as an induced subgraph.

$\left|\mathcal{H}_{6}\right|=34$. Since we do not know of any reasonable way to draw complicated 3 -graphs, we list the elements of $\mathcal{H}_{6}$ in Table 1 by their edge sets. The eight regular 3 -graphs are listed first and denoted in bold.

A type is a totally labeled admissible 3-graph $\sigma$, i.e., an admissible 3-graph $\sigma$ with $V(\sigma)=[k]$ for some $k \geq 0$ called the size of $\sigma$ and denoted by $|\sigma|$. 
TABLE 1

Admissible 3-graphs on 6 vertices.

\begin{tabular}{|c|c|}
\hline & \\
\hline$\overline{\mathbf{H}_{1}}$ & $\begin{array}{ll}(1,2,3),(1,2,5),(1,4,6),(2,4,6),(3,4,5),(3,5,6) \\
\end{array}$ \\
\hline $\mathrm{H}_{2}$ & $(1,2,3),(1,2,5),(1,2,6),(3,4,5),(3,4,6),(3,5,6),(4,5,6)$ \\
\hline$\overline{\mathbf{H}_{3}}$ & $(1,2,3),(1,2,5),(1,2,6),(1,3,6),(1,5,6),(2,3,6),(2,5,6),(3,4,5)$ \\
\hline $\mathrm{H}_{4}$ & $(1,2,3),(1,2,5),(1,3,5),(1,4,6),(2,3,5),(2,4,6),(3,4,6),(4,5,6)$ \\
\hline $\mathrm{H}_{5}$ & $(1,2,3),(1,2,5),(1,2,6),(1,3,5),(1,3,6),(1,5,6),(2,3,5),(2,3,6),(2,5,6),(3,5,6)$ \\
\hline$\overline{H_{6}}$ & $\begin{array}{l}(1,2,3),(1,4,5),(1,4,6),(1,5,6),(2,4,5),(2,4,6),(2,5,6),(3,4,5),(3,4,6),(3,5,6), \\
(4,5,6)\end{array}$ \\
\hline $\mathrm{H}_{7}$ & $\begin{array}{l}(1,2,3),(1,2,4),(1,2,5),(1,2,6),(1,3,5),(1,3,6),(1,4,5),(1,4,6),(1,5,6),(2,3,5), \\
(2,3,6),(2,4,5),(2,4,6),(2,5,6),(3,5,6),(4,5,6)\end{array}$ \\
\hline $\mathbf{H}_{8}$ & $\begin{array}{l}(1,2,3),(1,2,4),(1,2,5),(1,2,6),(1,3,4),(1,3,5),(1,3,6),(1,4,5),(1,4,6),(1,5,6), \\
(2,3,4),(2,3,5),(2,3,6),(2,4,5),(2,4,6),(2,5,6),(3,4,5),(3,4,6),(3,5,6),(4,5,6)\end{array}$ \\
\hline$H_{9}$ & $(1,2,3),(1,2,5),(1,2,6),(1,3,6),(2,3,6),(3,4,5),(4,5,6)$ \\
\hline$H_{10}$ & $(1,2,3),(1,2,5),(1,3,6),(1,4,6),(2,4,6),(2,5,6),(3,4,5)$ \\
\hline$H_{11}$ & $(1,2,3),(1,2,5),(1,3,6),(1,4,6),(2,4,6),(3,4,5),(3,5,6)$ \\
\hline$H_{12}$ & $(1,2,3),(1,2,5),(1,2,6),(1,3,6),(1,4,6),(2,3,6),(3,4,5),(4,5,6)$ \\
\hline$H_{13}$ & $(1,2,3),(1,2,5),(1,3,6),(1,4,5),(2,4,6),(2,5,6),(3,4,5),(3,4,6)$ \\
\hline$H_{14}$ & $(1,2,3),(1,2,5),(1,2,6),(1,4,6),(3,4,5),(3,4,6),(3,5,6),(4,5,6)$ \\
\hline$H_{15}$ & $(1,2,3),(1,2,5),(1,3,6),(1,4,6),(2,4,6),(2,5,6),(3,4,5),(3,5,6)$ \\
\hline$H_{16}$ & $(1,2,3),(1,2,5),(1,4,6),(2,4,6),(3,4,5),(3,4,6),(3,5,6),(4,5,6)$ \\
\hline$H_{17}$ & $(1,2,3),(1,2,5),(1,2,6),(1,3,6),(1,4,6),(1,5,6),(2,3,6),(2,5,6),(3,4,5)$ \\
\hline$H_{18}$ & $(1,2,3),(1,2,5),(1,2,6),(1,3,6),(1,5,6),(2,3,6),(2,5,6),(3,4,5),(3,4,6)$ \\
\hline$H_{19}$ & $(1,2,3),(1,2,5),(1,2,6),(1,3,6),(2,3,6),(3,4,5),(3,4,6),(3,5,6),(4,5,6)$ \\
\hline$H_{20}$ & $(1,2,3),(1,2,5),(1,2,6),(1,3,6),(1,4,5),(2,3,6),(2,4,6),(3,4,5),(3,5,6)$ \\
\hline$H_{21}$ & $(1,2,3),(1,2,5),(1,2,6),(1,3,6),(1,4,5),(2,3,6),(2,4,6),(3,4,5),(4,5,6)$ \\
\hline$H_{22}$ & $(1,2,3),(1,2,5),(1,3,6),(1,4,5),(1,4,6),(2,4,6),(2,5,6),(3,4,5),(3,5,6)$ \\
\hline$H_{23}$ & $(1,2,3),(1,2,5),(1,2,6),(1,3,6),(1,4,5),(1,5,6),(2,3,6),(2,4,6),(2,5,6),(3,4,5)$ \\
\hline $\mathrm{H}_{24}$ & $(1,2,3),(1,2,5),(1,2,6),(1,3,6),(1,4,5),(1,5,6),(2,3,6),(2,5,6),(3,4,5),(3,4,6)$ \\
\hline$H_{25}$ & $(1,2,3),(1,2,5),(1,2,6),(1,3,6),(1,4,5),(2,3,6),(3,4,5),(3,4,6),(3,5,6),(4,5,6)$ \\
\hline$H_{26}$ & $(1,2,3),(1,2,6),(1,3,6),(1,4,5),(1,4,6),(1,5,6),(2,3,6),(2,4,5),(3,4,5),(4,5,6)$ \\
\hline $\mathrm{H}_{27}$ & $(1,2,3),(1,2,4),(1,3,5),(1,4,6),(1,5,6),(2,3,6),(2,4,5),(2,5,6),(3,4,5),(3,4,6)$ \\
\hline$H_{28}$ & $\begin{array}{l}(1,2,3),(1,2,5),(1,2,6),(1,3,5),(1,3,6),(1,4,6),(1,5,6),(2,3,5),(2,3,6),(2,5,6), \\
(3,5,6)\end{array}$ \\
\hline$H_{29}$ & $\begin{array}{l}(1,2,3),(1,2,5),(1,2,6),(1,3,6),(1,4,5),(1,4,6),(1,5,6),(2,3,6),(2,5,6),(3,4,5), \\
(4,5,6)\end{array}$ \\
\hline$H_{30}$ & $\begin{array}{l}(1,2,3),(1,2,5),(1,2,6),(1,3,5),(1,3,6),(1,4,5),(1,5,6),(2,3,5),(2,3,6),(2,4,6), \\
(2,5,6),(3,5,6)\end{array}$ \\
\hline$H_{31}$ & $\begin{array}{l}(1,2,3),(1,2,4),(1,2,6),(1,3,5),(1,3,6),(1,4,6),(1,5,6),(2,3,6),(2,4,5),(2,4,6), \\
(3,4,5),(3,5,6)\end{array}$ \\
\hline$H_{32}$ & $\begin{array}{l}(1,2,3),(1,2,4),(1,2,5),(1,2,6),(1,3,5),(1,4,6),(2,3,5),(2,4,6),(3,4,5),(3,4,6), \\
(3,5,6),(4,5,6)\end{array}$ \\
\hline $\mathrm{H}_{33}$ & $\begin{array}{l}(1,2,3),(1,2,5),(1,2,6),(1,3,5),(1,3,6),(1,4,5),(1,4,6),(1,5,6),(2,3,5),(2,3,6), \\
(2,5,6),(3,5,6),(4,5,6)\end{array}$ \\
\hline$H_{34}$ & $\begin{array}{l}(1,2,3),(1,2,4),(1,2,5),(1,2,6),(1,3,5),(1,3,6),(1,4,6),(1,5,6),(2,3,5),(2,3,6), \\
(2,4,6),(2,5,6),(3,4,5),(3,5,6)\end{array}$ \\
\hline
\end{tabular}

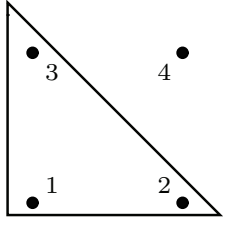

$\tau_{1}$

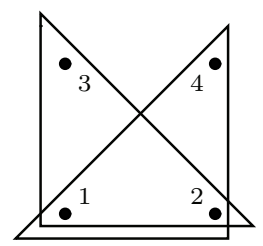

$\tau_{2}$

FIG. 2. Types.

For $k=0,1,2$, there is only one type of size $k$ that will be denoted simply by $k$.

Except for these trivial types, we will need only two types of size 4 that are based upon $G_{1}, G_{2}$ and called $\tau_{1}, \tau_{2}$, respectively (Figure 2).

For a type $\sigma$ of size $k$, a $\sigma$-flag is a pair $F=(H, \theta)$, where $H$ is an admissible 3 -graph and $\theta:[k] \longrightarrow V(H)$ is an injective function defining an induced embedding 

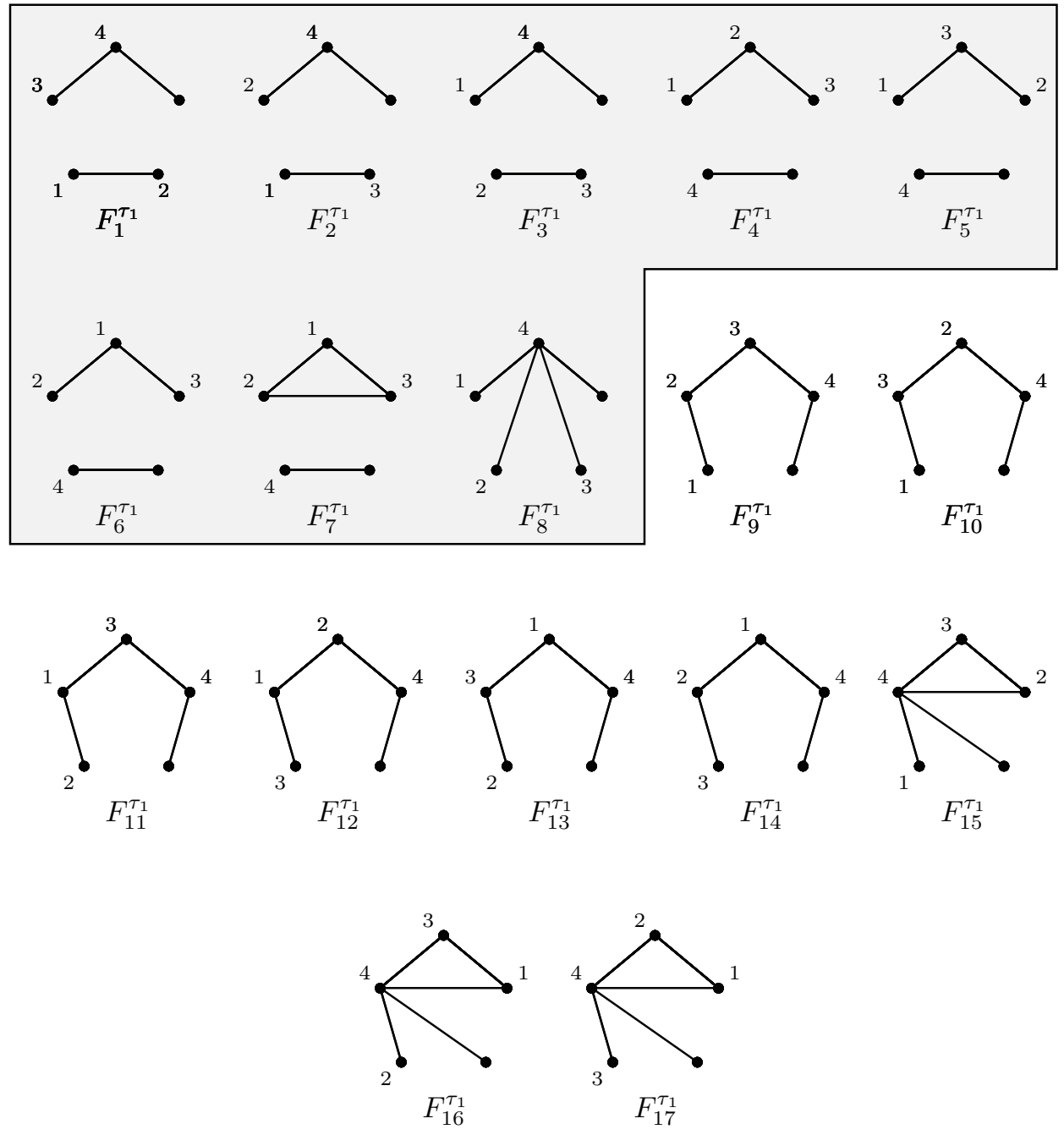

FIG. 3. $\mathcal{F}_{5}^{\tau_{1}}$.

of $\sigma$ into $H . \mathcal{F}^{\sigma}$ is the set of all $\sigma$-flags up to an isomorphism, and $\mathcal{F}_{\ell}^{\sigma} \subseteq \mathcal{F}^{\sigma}$ is the set of all $\sigma$-flags on $\ell$ vertices.

For any $\sigma, \mathcal{F}_{|\sigma|}^{\sigma}$ consists of the only element $(\sigma$, id), where id $: \sigma \longrightarrow \sigma$ is the identity mapping. This special flag is denoted by $1_{\sigma}$ or simply by 1 when $\sigma$ is clear from the context.

$\mathcal{F}_{\ell}^{0}$ is simply $\mathcal{H}_{\ell}$.

$\mathcal{F}_{3}^{1}$ consists of two elements: an edge and an independent set on 3 vertices, with one distinguished vertex each. We will denote the edge with one distinguished vertex by $e$.

$\left|\mathcal{F}_{5}^{\tau_{1}}\right|=17$ and $\left|\mathcal{F}_{5}^{\tau_{2}}\right|=15$. These flags are shown (using the same convention as on Figure 1) on Figures 3 and 4; a flag is called regular (and placed in the shaded area) iff its underlying admissible 3 -graph is so.

Given $F=(H, \theta) \in \mathcal{F}_{\ell}^{\sigma}$ and $F_{1} \in \mathcal{F}_{\ell_{1}}^{\sigma}$ with $\ell_{1} \leq \ell$, the (key) quantity $p\left(F_{1}, F\right) \in$ $[0,1]$ is defined as follows [22, Definition 1]. We choose in $V(H)$ uniformly at random a subset $\boldsymbol{V}_{\mathbf{1}}$ of size $\ell_{1}$ containing $\operatorname{im}(\theta)$ and let $p\left(F_{1}, F\right)$ be the probability that the 

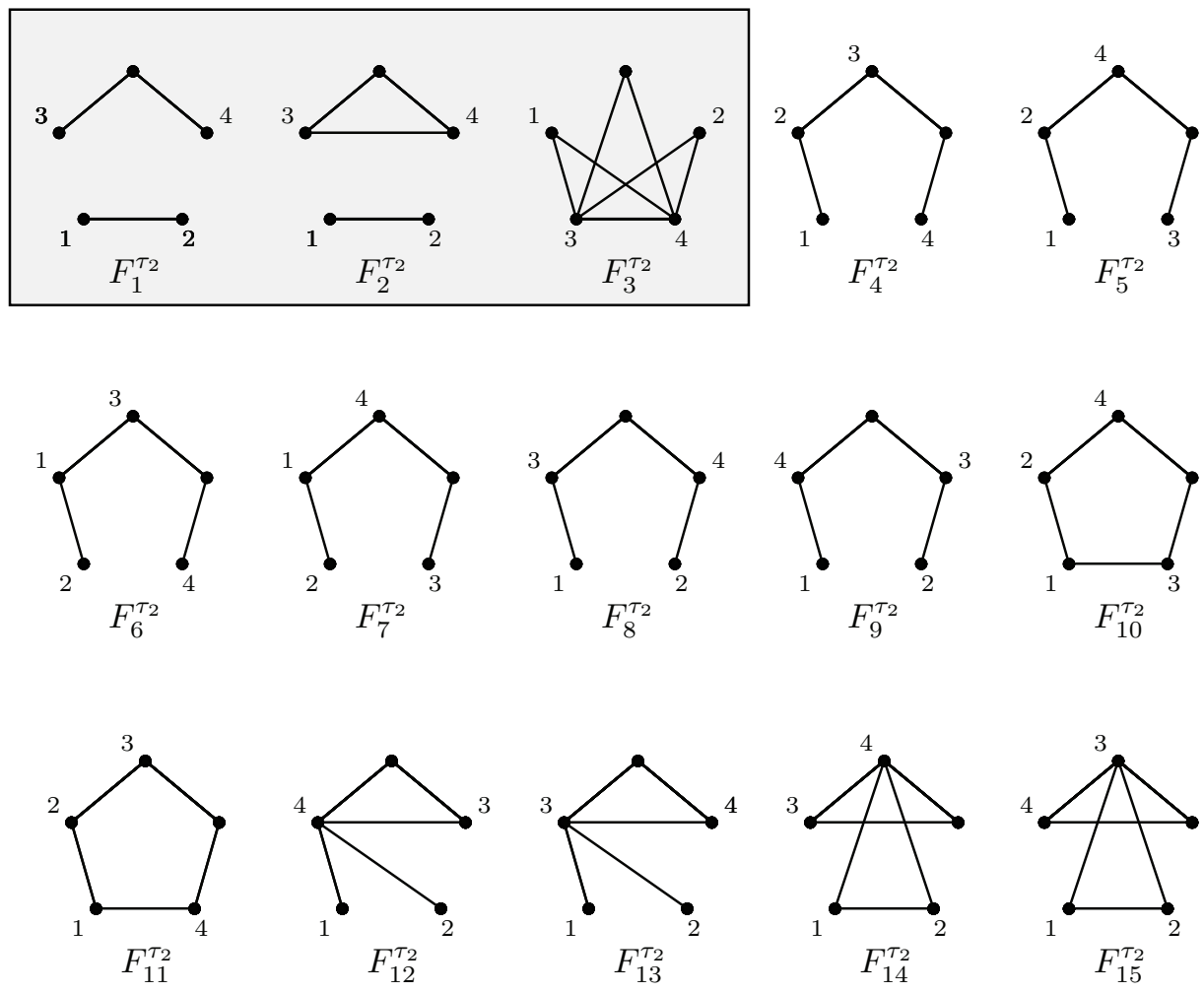

FIG. 4. $\mathcal{F}_{5}^{\tau_{2}}$.

induced $\sigma$-flag $\left.F\right|_{V_{1}}$ is isomorphic to $F_{1}$. The "chain rule" [22, Lemma 2.2] says that whenever $\ell_{1} \leq \tilde{\ell} \leq \ell$, we have the identity

$$
p\left(F_{1}, F\right)=\sum_{\widetilde{F} \in \mathcal{F}_{\bar{\ell}}^{\sigma}} p\left(F_{1}, \widetilde{F}\right) p(\widetilde{F}, F) .
$$

We consider the space $\mathbb{R} \mathcal{F}^{\sigma}$ of formal finite linear combinations of $\sigma$-flags with real coefficients; the elements $f=\sum_{\widetilde{F}} \alpha_{\widetilde{F}} \widetilde{F}$ should be thought of as "flag parameters" mapping sufficiently large flags $F$ to $p(f, F) \stackrel{\text { def }}{=} \sum_{\widetilde{F}} \alpha_{\widetilde{F}} p(\widetilde{F}, F)$. Identities (3) then suggest that it is natural to factor $\mathbb{R} \mathcal{F}^{\sigma}$ by the subspace $\mathcal{K}^{\sigma}$ of "identically zero flag parameters" generated by all elements of the form

$$
F_{1}-\sum_{\widetilde{F} \in \mathcal{F}_{\tilde{\ell}}^{\sigma}} p\left(F_{1}, \widetilde{F}\right) \widetilde{F},
$$

where $F_{1} \in \mathcal{F}_{\ell_{1}}^{\sigma}$ with $\ell_{1} \leq \tilde{\ell}$; denote $\mathbb{R} \mathcal{F}^{\sigma} / \mathcal{K}^{\sigma}$ by $\mathcal{A}^{\sigma}$. We also let $\mathcal{A}_{\ell}^{\sigma}$ be the subspace in $\mathcal{A}^{\sigma}$ generated by $\mathcal{F}_{\ell}^{\sigma}$. $\mathcal{A}^{\sigma}$ is naturally endowed with the structure of a commutative associative algebra [22, Lemma 2.4]. We will give the exact recipe for computing $F_{1} F_{2}$ only in conjunction with the averaging operator below, but the intuition behind this definition is that although the flag parameter $F \mapsto p\left(F_{1}, F\right) \cdot p\left(F_{2}, F\right)$ is in general different from $F \mapsto p\left(F_{1} \cdot F_{2}, F\right)$, this difference becomes negligible when the size of $F$ grows to infinity [22, Lemma 2.3]. Therefore, the "idealized" function $F \mapsto$ 
$p(F, \widehat{F})$ for "very large" $\widehat{F}$ should be expected to represent an algebra homomorphism $\phi \in \operatorname{Hom}\left(\mathcal{A}^{\sigma}, \mathbb{R}\right)$. Taking into account also the non-negativity property $p(F, \widehat{F}) \geq 0$, denote

$$
\operatorname{Hom}^{+}\left(\mathcal{A}^{\sigma}, \mathbb{R}\right) \stackrel{\text { def }}{=}\left\{\phi \in \operatorname{Hom}\left(\mathcal{A}^{\sigma}, \mathbb{R}\right) \mid \forall F \in \mathcal{F}^{\sigma} \phi(F) \geq 0\right\}
$$

Then it turns out that our intuition pays off and this object indeed captures all asymptotically true relations in extremal combinatorics. Referring the interested reader to [22, Corollary 3.4] for full details, in our particular situation we have

$$
\pi_{\min }\left(I_{4}^{3}, G_{3}\right)=\min _{\phi \in \operatorname{Hom}^{+}\left(\mathcal{A}^{0}, \mathbb{R}\right)} \phi(\rho) .
$$

The linear operator $\llbracket \cdot \rrbracket_{\sigma}: \mathcal{A}^{\sigma} \longrightarrow \mathcal{A}^{0}[22$, Section 2.2] corresponds to averaging. In order to understand its intuitive meaning, let $f \in \mathcal{A}^{\sigma}$, and let $H$ be a sufficiently large admissible 3 -graph. Generate a random injective mapping $\boldsymbol{\theta}$ from $[k]$ to $V(H)$ uniformly at random, and compute the expected value of $p(f,(H, \boldsymbol{\theta}))$, where we let $p(f,(H, \boldsymbol{\theta})) \stackrel{\text { def }}{=} 0$ if $\boldsymbol{\theta}$ does not define an embedding of $\sigma$ into $H$. Then the characteristic property of the element $\llbracket f \rrbracket_{\sigma} \in \mathcal{A}^{0}$ is that this expectation must be equal to $p\left(\llbracket f \rrbracket_{\sigma}, H\right)$ for any (sufficiently large) $H$.

After this little bit of theory, let us look at the computational aspect. For $F_{1} \in$ $\mathcal{F}_{\ell_{1}}^{\sigma}, F_{2} \in \mathcal{F}_{\ell_{2}}^{\sigma}$, the element $\llbracket F_{1} F_{2} \rrbracket_{\sigma}$ is computed as follows. Fix an arbitrary $\ell \geq$ $\ell_{1}+\ell_{2}-|\sigma|$ (the relations (4) will ensure that the final result does not depend on $\ell$; see the example below) and let $\llbracket F_{1} F_{2} \rrbracket_{\sigma} \stackrel{\text { def }}{=} \sum_{H \in \mathcal{H}_{\ell}} \alpha_{H} H$, where non-negative coefficients $\alpha_{H}$ are computed as follows. Choose uniformly at random an injective mapping $\boldsymbol{\theta}:[k] \longrightarrow V(H)$ and two subsets $\boldsymbol{V}_{\mathbf{1}}, \boldsymbol{V}_{\mathbf{2}} \subseteq V(H)$ of sizes $\ell_{1}, \ell_{2}$, respectively, subject to the only restriction $\boldsymbol{V}_{\mathbf{1}} \cap \boldsymbol{V}_{\mathbf{2}}=\operatorname{im}(\theta)$. Then $\alpha_{H}$ is the probability that $\boldsymbol{\theta}$ defines an embedding of $\sigma$ into $H$ and $\sigma$-flags $\left(\left.H\right|_{\boldsymbol{V}_{\mathbf{1}}}, \boldsymbol{\theta}\right),\left(\left.H\right|_{\boldsymbol{V}_{\mathbf{2}}}, \boldsymbol{\theta}\right)$ are isomorphic to $F_{1}, F_{2}$, respectively.

As an example, let us compute $\llbracket(e-4 / 9)(1-e) \rrbracket_{1}$ (this element will really be used in our proof). First,

$$
\llbracket(e-4 / 9)(1-e) \rrbracket_{1}=\llbracket-4 / 9+13 / 9 e-e^{2} \rrbracket_{1}=-\frac{4}{9}+\frac{13}{9} \rho-\llbracket e^{2} \rrbracket_{1} .
$$

Now we will express this element as a linear combination of hypergraphs in $\mathcal{H}_{6}$. By (4), $1=\sum_{i=1}^{34} H_{i}$ and $\rho=\sum_{i=1}^{34} \alpha_{i} H_{i}$, where $\alpha_{i}$ is the edge density of $H_{i}$. And by the above description, $\llbracket e^{2} \rrbracket_{1}=\frac{1}{90} \sum_{i=1}^{34} a_{i} H_{i}$, where $a_{i}$ is the number of unordered pairs $\left\{e_{1}, e_{2}\right\} \subseteq E\left(H_{i}\right)$ with $\left|e_{1} \cap e_{2}\right|=1$. The result of this computation is shown in Table 2 ; the interested reader may readily check that we will arrive at the same result if we first express $\llbracket(e-4 / 9)(1-e) \rrbracket_{1}$ as a linear combination of elements of $\mathcal{H}_{5}$ and then lift this expression to $\mathcal{H}_{6}$ using relations (4).

For $f, g \in \mathcal{A}^{0}$, we abbreviate $\forall \phi \in \operatorname{Hom}^{+}\left(\mathcal{A}^{0}, \mathbb{R}\right)(\phi(f) \geq \phi(g))$ to $f \geq g$. This is a partial preorder on $\mathcal{A}^{0}$, and we need to prove that $\rho \geq 4 / 9$. What we know [22, pages $1259-1260 \rrbracket$ is that $\llbracket f^{2} \rrbracket_{\sigma} \geq 0$ for every type $\sigma$ and every $f \in \mathcal{A}^{\sigma}$. This immediately implies that

$$
\llbracket Q\left(f_{1}, \ldots, f_{r}\right) \rrbracket_{\sigma} \geq 0,
$$

where $f_{1}, \ldots, f_{r} \in \mathcal{A}^{\sigma}$ and $Q$ is an arbitrary positive semidefinite quadratic form. 
TABLE 2

$$
\llbracket(e-4 / 9)(1-e) \rrbracket_{1} .
$$

\begin{tabular}{|c|c|c|c||c|c|c|c|}
\hline Name & $\rho$ & $\llbracket e^{2} \rrbracket_{1}$ & $\llbracket(e-4 / 9)(1-e) \rrbracket_{1}$ & Name & $\rho$ & $\llbracket e^{2} \rrbracket_{1}$ & $\llbracket(e-4 / 9)(1-e) \rrbracket_{1}$ \\
\hline \hline$H_{1}$ & $3 / 10$ & $2 / 15$ & $-13 / 90$ & $H_{18}$ & $9 / 20$ & $8 / 45$ & $1 / 36$ \\
\hline$H_{2}$ & $7 / 20$ & $1 / 10$ & $-7 / 180$ & $H_{19}$ & $9 / 20$ & $1 / 6$ & $7 / 180$ \\
\hline$H_{3}$ & $2 / 5$ & $2 / 15$ & 0 & $H_{20}$ & $9 / 20$ & $19 / 90$ & $-1 / 180$ \\
\hline$H_{4}$ & $2 / 5$ & $2 / 15$ & 0 & $H_{21}$ & $9 / 20$ & $1 / 5$ & $1 / 180$ \\
\hline$H_{5}$ & $1 / 2$ & $1 / 6$ & $1 / 9$ & $H_{22}$ & $9 / 20$ & $4 / 15$ & $-11 / 180$ \\
\hline$H_{6}$ & $11 / 20$ & $3 / 10$ & $1 / 20$ & $H_{23}$ & $1 / 2$ & $11 / 45$ & $1 / 30$ \\
\hline$H_{7}$ & $4 / 5$ & $3 / 5$ & $1 / 9$ & $H_{24}$ & $1 / 2$ & $7 / 30$ & $2 / 45$ \\
\hline$H_{8}$ & 1 & 1 & 0 & $H_{25}$ & $1 / 2$ & $2 / 9$ & $1 / 18$ \\
\hline$H_{9}$ & $7 / 20$ & $1 / 9$ & $-1 / 20$ & $H_{26}$ & $1 / 2$ & $2 / 9$ & $1 / 18$ \\
\hline$H_{10}$ & $7 / 20$ & $1 / 6$ & $-19 / 180$ & $H_{27}$ & $1 / 2$ & $1 / 3$ & $-1 / 18$ \\
\hline$H_{11}$ & $7 / 20$ & $1 / 6$ & $-19 / 180$ & $H_{28}$ & $11 / 20$ & $7 / 30$ & $7 / 60$ \\
\hline$H_{12}$ & $2 / 5$ & $7 / 45$ & $-1 / 45$ & $H_{29}$ & $11 / 20$ & $5 / 18$ & $13 / 180$ \\
\hline$H_{13}$ & $2 / 5$ & $1 / 5$ & $-1 / 15$ & $H_{30}$ & $3 / 5$ & $14 / 45$ & $1 / 9$ \\
\hline$H_{14}$ & $2 / 5$ & $13 / 90$ & $-1 / 90$ & $H_{31}$ & $3 / 5$ & $16 / 45$ & $1 / 15$ \\
\hline$H_{15}$ & $2 / 5$ & $19 / 90$ & $-7 / 90$ & $H_{32}$ & $3 / 5$ & $1 / 3$ & $4 / 45$ \\
\hline$H_{16}$ & $2 / 5$ & $7 / 45$ & $-1 / 45$ & $H_{33}$ & $13 / 20$ & $11 / 30$ & $23 / 180$ \\
\hline$H_{17}$ & $9 / 20$ & $17 / 90$ & $1 / 60$ & $H_{34}$ & $7 / 10$ & $7 / 15$ & $1 / 10$ \\
\hline
\end{tabular}

3. Proof of Theorem 1. The upper bound $\pi_{\min }\left(I_{4}^{3}, G_{3}\right) \leq 4 / 9$ is provided by Turán's construction, so we only have to prove the opposite inequality $\pi_{\min }\left(I_{4}^{3}, G_{3}\right) \geq$ $4 / 9$ or, in our terminology, that $\rho \geq 4 / 9$.

First note that

$$
\llbracket(e-4 / 9)(1-e) \rrbracket_{1}=\frac{5}{9} \llbracket e-4 / 9 \rrbracket_{1}-\llbracket(e-4 / 9)^{2} \rrbracket_{1} \leq_{\text {by }(6)} \frac{5}{9}(\rho-4 / 9) ;
$$

therefore, it is sufficient to prove that $\llbracket(e-4 / 9)(1-e) \rrbracket_{1} \geq 0$. And, again due to (6), it is sufficient to exhibit positive definite quadratic forms $Q_{1}\left(f_{1}, \ldots, f_{4}\right)\left(f_{i} \in \mathcal{A}_{5}^{\tau_{1}}\right)$ and $Q_{2}\left(g_{0}, g_{1}, \ldots, g_{5}\right)\left(g_{i} \in \mathcal{A}_{5}^{\tau_{2}}\right)$ such that

$$
\llbracket(e-4 / 9)(1-e) \rrbracket_{1} \geq \llbracket Q_{1}\left(f_{1}, \ldots, f_{4}\right) \rrbracket_{\tau_{1}}+\llbracket Q_{2}\left(g_{0}, \ldots, g_{5}\right) \rrbracket_{\tau_{2}} .
$$

As promised in the Introduction, here we present the bare result, deferring a little bit of intuition to the next section.

We let (cf. Figure 3)

$$
\begin{aligned}
& f_{1} \stackrel{\text { def }}{=}\left(F_{1}^{\tau_{1}}+F_{2}^{\tau_{2}}+F_{3}^{\tau_{3}}\right)-\left(F_{4}^{\tau_{1}}+F_{5}^{\tau_{1}}+F_{6}^{\tau_{1}}\right) ; \\
& f_{2} \stackrel{\text { def }}{=}\left(F_{1}^{\tau_{1}}+F_{2}^{\tau_{2}}+F_{3}^{\tau_{3}}\right)+2 F_{7}^{\tau_{1}}-F_{8}^{\tau_{1}} ; \\
& f_{3} \stackrel{\text { def }}{=} \sum_{i=9}^{14} F_{i}^{\tau_{1}} ; \\
& f_{4} \stackrel{\text { def }}{=} \sum_{i=15}^{17} F_{i}^{\tau_{1}} .
\end{aligned}
$$

The quadratic form $Q_{1}$ is represented by the matrix

$$
M_{1} \stackrel{\text { def }}{=} \frac{1}{24}\left(\begin{array}{cccc}
32 & -4 & -22 & 25 \\
-4 & 16 & 1 & -13 \\
-22 & 1 & 17 & -17 \\
25 & -13 & -17 & 27
\end{array}\right)
$$

its minimal eigenvalue is $0.01417 \ldots$ (note that the estimates on the eigenvalues of $M_{1}$ and $M_{2}$ are the only floating point parts of the entire proof). 
$Q_{2}$ has the form $\frac{1}{2} g_{0}^{2}+Q_{2}^{+}\left(g_{1}, \ldots, g_{5}\right)$. The element $g_{0}$ is computed as $\sum_{F \in \mathcal{F}_{5}^{\tau_{2}}} \alpha_{F} F$, where $\alpha_{F}$ is the number of edges in $F$ among $\{(1,3, v),(2,4, v)\}$ minus the number of edges among $\{(1,4, v),(2,3, v)\}$ ( $v$ is the only unlabeled vertex in $F$ ). Thus, $g_{0}=-F_{4}^{\tau_{2}}+F_{5}^{\tau_{2}}+F_{6}^{\tau_{2}}-F_{7}^{\tau_{2}}+2 F_{8}^{\tau_{2}}-2 F_{9}^{\tau_{2}}+2 F_{10}^{\tau_{2}}-2 F_{11}^{\tau_{2}}$; the reader better familiar with the language from [22] should also notice that $g_{0}=\sum_{\substack{i \in\{1,2\} \\ j \in\{3,4\}}}(-1)^{i+j} \pi^{\tau_{2},[i, j]}\left(e^{*}\right)$, where $e^{*} \in \mathcal{F}_{3}^{2}$ is an edge with two distinguished vertices. But we do not need this interpretation here.

Next,

$$
\begin{aligned}
& g_{1} \stackrel{\text { def }}{=} F_{1}^{\tau_{2}}-F_{2}^{\tau_{2}} ; \\
& g_{2} \stackrel{\text { def }}{=} F_{1}^{\tau_{2}}-F_{3}^{\tau_{2}} ; \\
& g_{3} \stackrel{\text { def }}{=} \sum_{i=4}^{7} F_{i}^{\tau_{2}} ; \\
& g_{4} \stackrel{\text { def }}{=} F_{8}^{\tau_{2}}+F_{9}^{\tau_{2}} ; \\
& g_{5} \stackrel{\text { def }}{=} F_{12}^{\tau_{2}}+F_{13}^{\tau_{2}},
\end{aligned}
$$

and $Q_{2}^{+}$is represented by the symmetric matrix

$$
M_{2} \stackrel{\text { def }}{=} \frac{1}{24}\left(\begin{array}{ccccc}
64 & -20 & 6 & -42 & 44 \\
-20 & 40 & 25 & -41 & -19 \\
6 & 25 & 28 & -55 & -1 \\
-42 & -41 & -55 & 131 & -18 \\
44 & -19 & -1 & -18 & 33
\end{array}\right)
$$

with the minimal eigenvalue $0.0325 \ldots$.

The inequality (8) is checked by representing both sides in the form $\sum_{i=1}^{34} \alpha_{i} H_{i}\left(H_{i}\right.$ $\in \mathcal{H}_{6}$ ) and comparing coefficients $\alpha_{i}$; our final Table 3 (in which the first column is copied from Table 1) represents intermediate steps in this computation.

This completes the proof of Theorem 1.

4. Some reflections. Although the proof of Theorem 1 was mostly done on a computer, still there are a few important mathematical principles that have simplified both this work and the subsequent process of writing down its results. Like virtually anything in the theory of flag algebras, these principles are nothing else but a somewhat cleaned and mathematically structured form of certain intuition well known in the field.

Given a type $\sigma$, let $\Gamma_{\sigma}$ be its group of automorphisms (for example, $\Gamma_{\tau_{1}}=S_{3}$ and $\Gamma_{\tau_{2}}=\mathbb{Z}_{2} \oplus \mathbb{Z}_{2}$ ). Then $\Gamma_{\sigma}$ naturally acts on $\mathcal{A}^{\sigma}$ (by relabeling flags). This gives us a decomposition of $\mathcal{A}^{\sigma}$ as a $\Gamma_{\sigma}$-module in the form

$$
\mathcal{A}^{\sigma}=\mathcal{A}^{\sigma,+} \oplus \mathcal{A}^{\sigma,-},
$$

where $\mathcal{A}^{\sigma,+}$ consists of all $\Gamma_{\sigma}$-invariant elements, and $\mathcal{A}^{\sigma,-} \stackrel{\text { def }}{=}\left\{f \in \mathcal{A}^{\sigma} \mid \sum_{\gamma \in \Gamma_{\sigma}} \gamma f=\right.$ $0\}$. It is easy to see that $\llbracket \mathcal{A}^{\sigma,+} \mathcal{A}^{\sigma,-} \rrbracket_{\sigma}=0$. Hence in any application of the CauchySchwarz method, we may always assume that the quadratic form $Q$ in (6) is split into the sum of its invariant part $Q^{+}\left(\mathcal{A}^{\sigma,+}\right)$ and "anti-invariant" part $Q^{-}\left(\mathcal{A}^{\sigma,-}\right)$. This observation alone greatly reduces the dimension of the search space. In our example, $Q_{1}$ and $Q_{2}^{+}$are invariant, and $g_{0}^{2}$ is anti-invariant. 
TABLE 3

Final computation.

\begin{tabular}{|c|c|c|c|c|c|}
\hline Name & $\llbracket(e-4 / 9)(1-e) \rrbracket_{1}$ & $\llbracket g_{0}^{2} \rrbracket_{\tau_{2}}$ & $\llbracket Q_{1} \rrbracket \tau_{1}$ & $\llbracket Q_{2} \rrbracket_{\tau_{2}}$ & $\llbracket(e-4 / 9)(1-e) \rrbracket_{1}-\frac{1}{2} \llbracket g_{0}^{2} \rrbracket_{\tau_{2}}-\llbracket Q_{1} \rrbracket_{\tau_{1}}-\llbracket Q_{2} \rrbracket_{\tau_{2}}$ \\
\hline$\overline{H_{1}}$ & $\begin{aligned}-13 / 90 \\
\end{aligned}$ & 0 & $-7 / 30$ & $4 / 45$ & 0 \\
\hline $\mathrm{H}_{2}$ & $-7 / 180$ & $\overline{0}$ & $1 / 45$ & $-11 / 180$ & $\overline{0}$ \\
\hline$H_{3}$ & 0 & 0 & $1 / 36$ & $-1 / 36$ & 0 \\
\hline$H_{4}$ & 0 & 0 & $-8 / 45$ & $8 / 45$ & 0 \\
\hline $\mathrm{H}_{5}$ & $1 / 9$ & 0 & $1 / 9$ & 0 & 0 \\
\hline$H_{6}$ & $1 / 20$ & 0 & $2 / 15$ & $-1 / 12$ & 0 \\
\hline $\mathrm{H}_{7}$ & $1 / 9$ & 0 & 0 & $1 / 9$ & 0 \\
\hline$H_{8}$ & 0 & 0 & 0 & 0 & 0 \\
\hline$H_{9}$ & $-1 / 20$ & 0 & $-77 / 720$ & $19 / 360$ & $1 / 240$ \\
\hline$H_{10}$ & $-19 / 180$ & $-1 / 30$ & $-1 / 48$ & $-11 / 144$ & $1 / 120$ \\
\hline$H_{11}$ & $-19 / 180$ & $-1 / 15$ & $-9 / 160$ & $-1 / 30$ & $5 / 288$ \\
\hline$H_{12}$ & $-1 / 45$ & $1 / 45$ & $-103 / 14404$ & $-19 / 2160$ & $203 / 4320$ \\
\hline$H_{13}$ & $-1 / 15$ & $-1 / 10$ & $17 / 240$ & $-41 / 360$ & $19 / 720$ \\
\hline$H_{14}$ & $-1 / 90$ & $2 / 45$ & $-131 / 1440$ & $73 / 2160$ & $103 / 4320$ \\
\hline$H_{15}$ & $-7 / 90$ & $-1 / 5$ & $1 / 40$ & $-1 / 40$ & $1 / 45$ \\
\hline$H_{16}$ & $-1 / 45$ & 0 & $-1 / 360$ & $\begin{array}{l}-1 / 45 \\
\end{array}$ & $1 / 360$ \\
\hline$H_{17}$ & $1 / 60$ & 0 & $1 / 15$ & $-23 / 432$ & $7 / 2160$ \\
\hline$H_{18}$ & $1 / 36$ & $1 / 90$ & $11 / 288$ & $-13 / 360$ & $29 / 1440$ \\
\hline$H_{19}$ & $7 / 180$ & 0 & $-1 / 40$ & $11 / 360$ & $1 / 30$ \\
\hline$H_{20}$ & $-1 / 180$ & $1 / 30$ & $17 / 1440$ & $-7 / 180$ & $7 / 1440$ \\
\hline$H_{21}$ & $1 / 180$ & $4 / 45$ & $-23 / 480$ & $-7 / 1080$ & $67 / 4320$ \\
\hline $\mathrm{H}_{22}$ & $-11 / 180$ & $-2 / 5$ & $17 / 480$ & 0 & $149 / 1440$ \\
\hline$H_{23}$ & $1 / 30$ & $1 / 90$ & $-1 / 48$ & $-43 / 1080$ & $191 / 2160$ \\
\hline $\mathrm{H}_{24}$ & $2 / 45$ & $2 / 45$ & $1 / 144$ & $-43 / 1080$ & $119 / 2160$ \\
\hline $\mathrm{H}_{25}$ & $1 / 18$ & $2 / 45$ & $-17 / 360$ & \begin{tabular}{ll|}
$41 / 540$ \\
\end{tabular} & $1 / 216$ \\
\hline$H_{26}$ & $1 / 18$ & 0 & $-13 / 180$ & $4 / 135$ & $53 / 540$ \\
\hline $\mathrm{H}_{27}$ & $-1 / 18$ & $-2 / 3$ & 0 & 0 & $5 / 18$ \\
\hline $\mathrm{H}_{28}$ & $7 / 60$ & 0 & $47 / 720$ & $11 / 240$ & $1 / 180$ \\
\hline $\mathrm{H}_{29}$ & $13 / 180$ & 0 & $-17 / 1440$ & $17 / 720$ & $29 / 480$ \\
\hline$H_{30}$ & $1 / 9$ & 0 & $3 / 40$ & $11 / 360$ & $1 / 180$ \\
\hline$H_{31}$ & $1 / 15$ & $2 / 45$ & 0 & 0 & $2 / 45$ \\
\hline $\mathrm{H}_{32}$ & $4 / 45$ & 0 & 0 & 0 & $4 / 45$ \\
\hline$H_{33}$ & $23 / 180$ & 0 & $9 / 160$ & $19 / 360$ & $3 / 160$ \\
\hline$H_{34}$ & $1 / 10$ & 0 & 0 & 0 & $1 / 10$ \\
\hline
\end{tabular}

In order to reduce the dimension even further, suppose that our problem comes equipped with a set $\Phi \subseteq \operatorname{Hom}^{+}\left(\mathcal{A}^{0}, \mathbb{R}\right)$ of "conjectured extremal homomorphisms" (in our case consisting of the single homomorphism $\phi_{T}$ corresponding to Turán's construction). Denote by $\Delta^{\sigma}$ the ideal in $\mathcal{A}^{\sigma}$ consisting of all relations that are "identically true" on all $\phi \in \Phi$. For $|\sigma|>0$ this is rigorously defined (using the apparatus from [22, Section 3.2]) as $\Delta^{\sigma} \stackrel{\text { def }}{=}\left\{f \in \mathcal{A}^{\sigma} \mid \forall \phi \in \Phi\left(\phi(\sigma)>0 \Longrightarrow \mathbf{P}\left[\phi^{\sigma}(f)=0\right]=1\right)\right\}$, but in every individual case this should be intuitively clear. These ideals behave well w.r.t. various operations on flag algebras; for example, $\llbracket \Delta^{\sigma} \rrbracket_{\sigma} \subseteq \Delta^{0}$. Then, since the desired semidefinite relation like (8) should have no slackness when it is evaluated by any extremal $\phi \in \Phi$, the quadratic forms $Q$ must also be supported on the ideals $\Delta^{\sigma}$. And since the ideal $\Delta^{\sigma}$ itself is a $\Gamma_{\sigma^{-}}$-submodule, this observation combines well with the one made above. Namely, $\Delta^{\sigma}=\Delta^{\sigma,+} \oplus \Delta^{\sigma,-}$, where $\Delta^{\sigma, *} \stackrel{\text { def }}{=} \Delta^{\sigma} \cap \mathcal{A}^{\sigma, *}$, and, therefore, we may always additionally assume that $Q^{+}$is supported on $\Delta^{\sigma,+}$ and that $Q^{-}$is supported on $\Delta^{\sigma,-}$.

Finally (as exemplified by the first eight entries in the last column of Table 3), the inequalities we are looking for should not have any slackness on regular (meaning $\exists \phi \in \Phi(\phi(H)>0))$ models $H$.

In our particular setting these general principles were combined with a computer search roughly as follows.

The choice of $\tau_{1}, \tau_{2}$ comes entirely from computer experiments - the analogue of (8) does not exist if we concentrate on $\tau_{1}, \tau_{4}$ or $\tau_{2}, \tau_{4}$. 
After the decision to restrict to $\tau_{1}, \tau_{2}$ has been made, the right-hand side of (8) will necessarily vanish on the (regular!) complete 3 -graph $H_{8}$, and, by the third principle, so must the left-hand side. Introducing the term $(1-e)$ into it is the simplest way to make it happen.

The choice of $g_{0}$ and the decision to avoid the anti-invariant part $Q^{\tau_{1},-}$ again completely belongs to the computer.

$\Delta^{\sigma}$ contains all singular $\sigma$-flags by definition. The remaining (regular) part of $\Delta^{\tau_{1},+}$ is generated by $f_{1}, f_{2}$, and the regular part of $\Delta^{\tau_{2},+}$ is generated by $g_{1}, g_{2}$. Therefore, both $\llbracket\left(\Delta_{5}^{\tau_{1},+}\right)^{2} \rrbracket_{\tau_{1}}$ and $\llbracket\left(\Delta_{5}^{\tau_{2},+}\right)^{2} \rrbracket_{\tau_{2}}\left(\Delta_{\ell}^{\sigma, *} \stackrel{\text { def }}{=} \Delta^{\sigma, *} \cap \mathcal{A}_{\ell}^{\sigma}\right)$ have dimension $\leq 3$ modulo the ideal $\Delta_{s}^{0} \subseteq \Delta^{0}$ generated by singular admissible 3 -graphs. It turns out that these subspaces have dimension exactly 3 each, and that moreover they are in general position mod $\Delta_{s}^{0}$. This completely determines the upper left $(2 \times 2)$ minors in the matrices $M_{1}, M_{2}$, since, once more, (8) must turn into an equality $\bmod \Delta_{s}^{0}$.

The rest of the proof entirely belongs to the computer; in particular, all other entries in $M_{1}, M_{2}$ are simply sufficiently close rational approximations to the outcome of a numerical SDP computation.

5. Conclusion and open problems. It immediately follows from definitions that $f \geq 0\left(f \in \mathcal{A}^{\sigma}\right)$ implies that for any $\epsilon>0$ there exists $\ell>0$ such that all the coefficients in the expansion

$$
(f+\epsilon)=\sum_{F \in \mathcal{F}_{\ell}^{\sigma}} p(f+\epsilon, F) F
$$

are positive and, thus, inequality $f \geq-\epsilon$ possesses a trivial proof in any reasonable sense. The complexity of this straightforward proof, however, grows when $\epsilon$ decreases.

The most interesting (in our opinion) general open question about asymptotic extremal combinatorics is whether any true relation $f \geq 0$ can be itself proved using a "finite amount of manipulation with finitely many flags." In [22, section 6] (see also [13, Problem 17]) we tried to present several precise refinements of this question. The current work gives one more example of an argument that can be formalized within the weakest of these refinements [22, Question 0 on page 1280]. This also solves Problem 19 from [13].

That would be very nice to give a purely combinatorial proof of our result, e.g., by finding an inequality like (8) with a combinatorial (as opposed to brute-force) verification algorithm.

Is Turán's configuration $\phi_{T}$ the only element of $\operatorname{Hom}^{+}\left(\mathcal{A}^{0}, \mathbb{R}\right)$ attaining the extremal value $\phi(\rho)=4 / 9$ ? Note that since all entries in the last column of Table 3 corresponding to singular 3-graphs are strictly positive, we have also proved that any extremal $\phi$ must satisfy $\forall H \in \mathcal{H}_{6}\left(\phi_{T}(H)=0 \Longrightarrow \phi(H)=0\right)$. This makes our uniqueness conjecture even more plausible.

And, of course, the problem of proving (or disproving) that $\pi_{\min }\left(I_{4}^{3}\right)=4 / 9$ remains one of the greatest challenges in the area.

\section{Recent developments.}

6.1. Other papers. After this paper was disseminated, Pikhurko [21] completely proved the uniqueness conjecture stated above. In fact, his methods allowed him to obtain a complete characterization of all extremal graphs for sufficiently large $n$.

Hladký, Král', and Norine [11] used the theory of flag algebras in their work on the Cacceta-Håggkvist conjecture. They showed that every digraph on $n$ vertices with minimum outdegree $0.3465 n$ contains an oriented triangle that significantly improves upon all previously known bounds. 
TABLE 4

Admissible 3-graphs on 7 vertices.

\begin{tabular}{|c|c|}
\hline \multicolumn{2}{|c|}{\begin{tabular}{l|l|l} 
me & Edge set \\
\end{tabular}} \\
\hline$\overline{H_{1}}$ & $\begin{array}{l}(1,2,3),(1,2,4),(1,2,5),(1,2,6),(1, \\
(2,3,4),(2,3,5),(2,4,6),(2,5,7),(2,\end{array}$ \\
\hline $\mathrm{H}_{2}$ & $\begin{array}{l}(1,2,3),(1,2,4),(1,2,5),(1,2,6),(1,3,4),(1,3,5),(1,3,7),(1,4,6),(1,5,7),(2,3,4), \\
(2,3,5),(2,4,6),(2,4,7),(2,6,7),(3,5,6),(3,5,7),(3,6,7),(4,5,6),(4,5,7),(4,6,7), \\
(5,6,7)\end{array}$ \\
\hline $\mathrm{H}_{3}$ & $\begin{array}{l}(1,2,3),(1,2,4),(1,2,5),(1,2,6),(1,2,7),(1,3,4),(1,3,5),(1,3,6),(1,4,5),(1,6,7), \\
(2,3,4),(2,3,5),(2,3,6),(2,4,5),(2,6,7),(3,4,5),(3,4,7),(3,5,7),(4,5,6),(4,5,7), \\
(4,6,7),(5,6,7)\end{array}$ \\
\hline $\mathrm{H}_{4}$ & $\begin{array}{l}(1,2,3),(1,2,4),(1,2,5),(1,2,6),(1,2,7),(1,3,4),(1,3,5),(1,3,6),(1,4,5),(1,4,6) \\
(1,5,7),(2,3,4),(2,3,5),(2,3,6),(2,4,5),(2,4,6),(2,5,7),(3,4,5),(3,4,6),(3,4,7) \\
(3,6,7),(4,6,7),(5,6,7)\end{array}$ \\
\hline$H_{5}$ & $\begin{array}{l}(1,2,3),(1,2,4),(1,2,5),(1,2,6),(1,2,7),(1,3,4),(1,3,5),(1,3,6),(1,3,7),(1,4,5), \\
(1,6,7),(2,3,4),(2,3,5),(2,3,6),(2,3,7),(2,4,5),(2,6,7),(3,4,5),(3,6,7),(4,5,6), \\
(4,5,7),(4,6,7),(5,6,7)\end{array}$ \\
\hline$H_{6}$ & $\begin{array}{l}(1,2,3),(1,2,4),(1,2,5),(1,2,6),(1,2,7),(1,3,4),(1,3,5),(1,3,6),(1,3,7),(1,4,5) \\
(1,4,6),(1,5,7),(2,3,4),(2,3,5),(2,3,6),(2,3,7),(2,4,5),(2,4,6),(2,5,7),(3,4,5) \\
(3,4,6),(3,5,7),(4,6,7),(5,6,7)\end{array}$ \\
\hline$H_{7}$ & $\begin{array}{l}(1,2,3),(1,2,4),(1,2,5),(1,2,6),(1,2,7),(1,3,4),(1,3,5),(1,3,6),(1,3,7),(1,4,5), \\
(1,4,6),(1,5,6),(2,3,4),(2,3,5),(2,3,6),(2,3,7),(2,4,5),(2,4,6),(2,5,6),(3,4,5), \\
(3,4,6),(3,5,6),(4,5,6),(4,5,7),(4,6,7),(5,6,7)\end{array}$ \\
\hline $\mathrm{H}_{8}$ & $\begin{array}{l}(1,2,3),(1,2,4),(1,2,5),(1,2,6),(1,2,7),(1,3,4),(1,3,5),(1,3,6),(1,3,7),(1,4,5) \\
(1,4,6),(1,4,7),(1,5,6),(2,3,4),(2,3,5),(2,3,6),(2,3,7),(2,4,5),(2,4,6),(2,4,7) \\
(2,5,6),(3,4,5),(3,4,6),(3,4,7),(3,5,6),(4,5,6),(5,6,7)\end{array}$ \\
\hline$H_{9}$ & $\begin{array}{l}(1,2,3),(1,2,4),(1,2,5),(1,2,6),(1,2,7),(1,3,4),(1,3,5),(1,3,6),(1,3,7),(1,4,5), \\
(1,4,6),(1,4,7),(1,5,6),(1,5,7),(2,3,4),(2,3,5),(2,3,6),(2,3,7),(2,4,5),(2,4,6), \\
(2,4,7),(2,5,6),(2,5,7),(3,4,5),(3,4,6),(3,4,7),(3,5,6),(3,5,7),(4,5,6),(4,5,7)\end{array}$ \\
\hline$\overline{H_{10}}$ & $\begin{array}{l}(1,2,3),(1,2,4),(1,2,5),(1,2,6),(1,2,7),(1,3,4),(1,3,5),(1,3,6),(1,3,7),(1,4,5) \\
(1,4,6),(1,4,7),(1,5,6),(1,5,7),(1,6,7),(2,3,4),(2,3,5),(2,3,6),(2,3,7),(2,4,5) \\
(2,4,6),(2,4,7),(2,5,6),(2,5,7),(2,6,7),(3,4,5),(3,4,6),(3,4,7),(3,5,6),(3,5,7) \\
(3,6,7),(4,5,6),(4,5,7),(4,6,7),(5,6,7)\end{array}$ \\
\hline
\end{tabular}

6.2. Mubayi challenge. Dhruv Mubayi (personal communication) compiled a list of a few other exact results, both known and new, about the density of 3-graphs similar to the one considered in the main body of our paper. As it has turned out, all of them can be re-proved with our method.

We utilize all our previous notation, with the exception that the notion of "admissible" will depend on the context.

We also let $E, N$ be the types of size 3 based on an edge (nonedge, respectively). We adopt the following enumerating scheme for $\mathcal{F}_{4}^{\sigma}(\sigma \in\{E, N\})$ : for $S \subseteq[3]$, $F_{S}^{\sigma} \in \mathcal{F}_{4}^{\sigma}$ is obtained from $\sigma$ by adding a new vertex $x$ with the link $\{[3] \backslash\{i\} \mid i \in S\}$.

We let $H_{5, i} \in \mathcal{H}_{5}(1 \leq i \leq 9)$ denote the $i$ th 3 -graph on Figure 1 . $H_{5,7}$ will be of particular interest to us; it will be denoted by $C_{5}$ and called the pentagon.

We begin with one old result by Frankl and Füredi [9]. Even if the structure of admissible graphs in this case is very restrictive, for some unclear reasons it requires substantially more computations than the others. We include our proof for the sake of completeness anyway, but the reader who does not want to get a wrong impression may want to skip to Theorem 3.

Theorem 2 (Frankl-Füredi). $\pi_{\min }\left(I_{4}^{3}, G_{1}, G_{3}\right)=13 / 18$.

Proof. For the lower bound see the original paper [9].

There are only 10 admissible graphs on 7 vertices listed in Table 4 . We have

$$
\begin{aligned}
& \llbracket(e-13 / 18)(1-e)(e+5 / 18) \rrbracket_{1} \\
& =\frac{1}{11340}\left(-835 H_{1}-378 H_{2}-313 H_{3}-92 H_{4}-300 H_{5}-247 H_{6}\right. \\
& \left.+675 H_{7}+248 H_{8}+575 H_{9}\right) .
\end{aligned}
$$




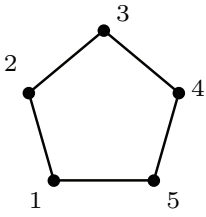

$\sigma_{1}$

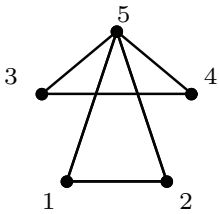

$\sigma_{2}$

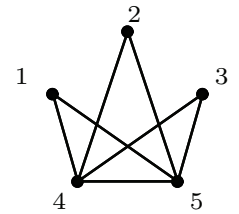

$\sigma_{3}$

FIG. 5. Some types of size 5 .

There are three ways to turn the pentagon $C_{5}$ into an $N$-flag (differing only by permuting labels), and we denote by $\mu_{5}^{N}\left(C_{5}\right)$ their sum; this notation is borrowed from [22, Section 4.3]. Let

$$
Q_{N} \stackrel{\text { def }}{=}\left(\mu_{5}^{N}\left(C_{5}\right)-1 / 6\right)^{2}
$$

then

$$
\llbracket Q_{N} \rrbracket_{N}=\frac{1}{1260}\left(25 H_{1}+112 H_{2}-3 H_{3}-28 H_{4}+12 H_{5}-19 H_{6}+9 H_{7}+8 H_{8}+5 H_{9}\right) .
$$

Let now $\sigma_{1}, \sigma_{2}, \sigma_{3}$ be the types of size 5 obtained from 3-graphs on Figure 1 as shown on Figure 5. Let $F_{1}^{\sigma_{1}} \in \mathcal{F}_{6}^{\sigma_{1}}$ be the flag obtained from $\sigma_{1}$ by adding a new vertex $x$ with the link $\{(1,2),(1,3),(2,3),(1,4),(1,5),(4,5),(2,5)\}$. The cyclic group $\mathbb{Z} \leq \Gamma_{\sigma_{1}}$ acts on $\mathcal{F}_{6}^{\sigma_{1}}$ by rotating the labels around the pentagon; let $\gamma \stackrel{\text { def }}{=}(12345)$ be its generator. This gives us four more flags $F_{2}^{\sigma_{1}}, F_{3}^{\sigma_{1}}, F_{4}^{\sigma_{1}}, F_{5}^{\sigma_{1}}$, where $F_{i} \stackrel{\text { def }}{=} \gamma^{i-1} F_{1}$. We now consider the quadratic form

$$
Q_{\sigma_{1}} \stackrel{\text { def }}{=} 2 \sum_{i \in[5]}\left(F_{i}^{\sigma_{1}}\right)^{2}+\sum_{|i-j|=1 \bmod 5} F_{i}^{\sigma_{1}} F_{j}^{\sigma_{1}}-3 \sum_{|i-j|=2 \bmod 5} F_{i}^{\sigma_{1}} F_{j}^{\sigma_{1}} .
$$

It is straightforward to check that $Q_{\sigma_{1}}$ is positive semidefinite, and

$$
\llbracket Q_{\sigma_{1}} \rrbracket_{\sigma_{1}}=\frac{1}{504}\left(-21 H_{2}-12 H_{3}+4 H_{4}+12 H_{6}\right) .
$$

$\mathcal{F}_{6}^{\sigma_{2}}$ is shown in Figure 6; the bold edge between $i$ and $j$ corresponds to the element $(x, i, j)$ of the link of the newly added vertex $x$. We let

$$
Q_{\sigma_{2}} \stackrel{\text { def }}{=}\left(F_{1}^{\sigma_{2}}+F_{2}^{\sigma_{2}}-F_{3}^{\sigma_{2}}-F_{4}^{\sigma_{2}}+2 F_{6}^{\sigma_{2}}-2 F_{7}^{\sigma_{2}}\right)^{2} ;
$$

then

$$
\llbracket Q_{\sigma_{2}} \rrbracket_{\sigma_{2}}=\frac{1}{315}\left(5 H_{1}-7 H_{2}-8 H_{3}-10 H_{4}+12 H_{6}-36 H_{7}+24 H_{8}\right) .
$$

$\mathcal{F}_{6}^{\sigma_{3}}$ is shown in Figure 7. Let

$$
Q_{\sigma_{3}}^{+} \stackrel{\text { def }}{=}\left(F_{9}^{\sigma_{3}}-1 / 3\right)^{2}
$$

then

$$
\llbracket Q_{\sigma_{3}}^{+} \rrbracket_{\sigma_{3}}=\frac{1}{1890}\left(5 H_{1}+7 H_{2}+6 H_{3}-4 H_{4}+4 H_{5}-15 H_{6}+6 H_{7}-16 H_{8}+40 H_{9}\right) .
$$



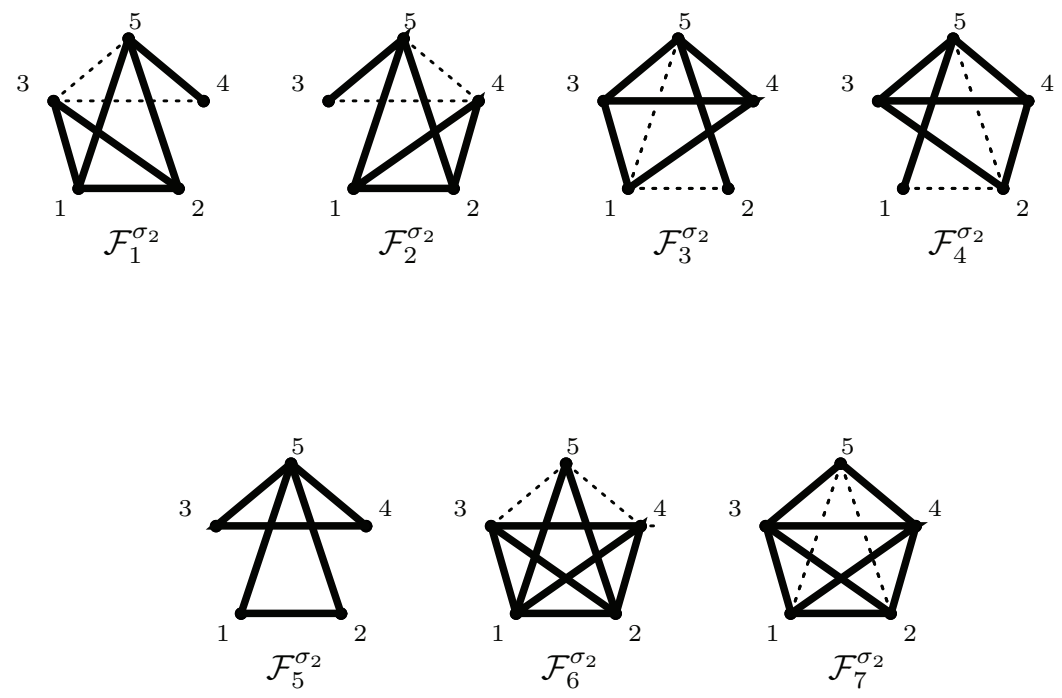

FIG. 6. $\mathcal{F}_{6}^{\sigma_{2}}$.
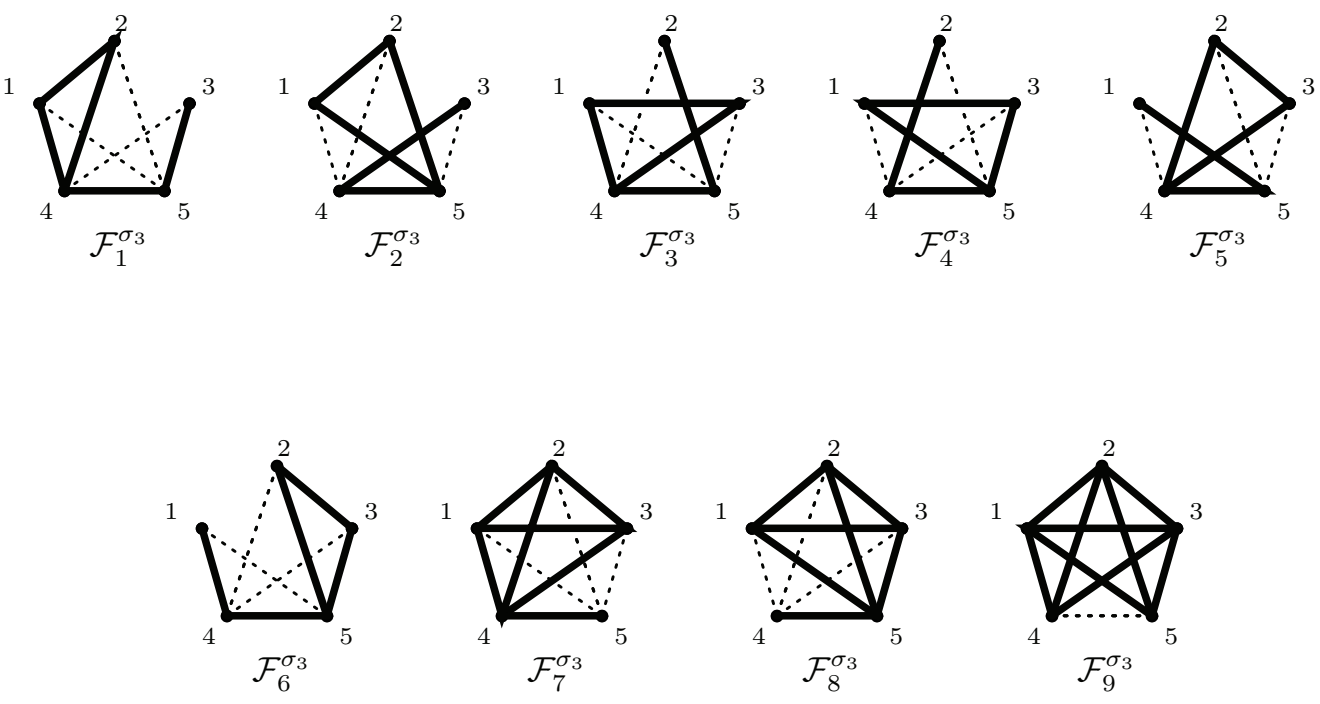

FIG. $7 . \mathcal{F}_{6}^{\sigma_{3}}$.

And finally we define

$$
\begin{aligned}
& h_{1} \stackrel{\text { def }}{=} F_{1}^{\sigma_{3}}-F_{2}^{\sigma_{3}}+F_{3}^{\sigma_{3}}-F_{4}^{\sigma_{3}}+F_{5}^{\sigma_{3}}-F_{6}^{\sigma_{3}}, \\
& h_{2} \stackrel{\text { def }}{=} F_{7}^{\sigma_{3}}-F_{8}^{\sigma_{3}}, \\
& Q_{\sigma_{3}}^{-} \stackrel{\text { def }}{=} \frac{143}{27} h_{1}^{2}+\frac{85}{18} h_{1} h_{2}+\frac{8}{3} h_{2}^{2} .
\end{aligned}
$$

Then $Q_{\sigma_{3}}^{-}$is positive semidefinite, and

$$
\llbracket Q_{\sigma_{3}}^{-} \rrbracket_{\sigma_{3}}=\frac{1}{5670}\left(-715 H_{1}-1001 H_{2}+31 H_{3}+255 H_{4}-288 H_{5}-72 H_{6}+432 H_{7}\right) .
$$


Finally, combining pieces together, we get

$$
\begin{aligned}
& \llbracket(e-13 / 18)(1-e)(e+5 / 18) \rrbracket_{1} \\
& \quad=\frac{19}{9} \llbracket Q_{N} \rrbracket_{N}+\frac{49}{45} \llbracket Q_{\sigma_{1}} \rrbracket_{\sigma_{1}}+\frac{1}{3} \llbracket Q_{\sigma_{2}} \rrbracket_{\sigma_{2}}+2 \llbracket Q_{\sigma_{3}}^{+} \rrbracket_{\sigma_{3}}+\llbracket Q_{\sigma_{3}}^{-} \rrbracket_{\sigma_{3}}+\frac{1}{1080} h_{2} \geq 0 .
\end{aligned}
$$

Similar to (7), $\rho \geq 13 / 18$ follows by summing this inequality with $\llbracket e(e-13 / 18)^{2} \rrbracket_{1} \geq 0$.

Theorem 3 (Mubayi, unpublished). $\pi_{\min }\left(I_{4}^{3}, G_{1}, G_{3}, C_{5}\right)=7 / 9$.

Proof. For the lower bound (Mubayi) fix again an almost balanced function $\chi: V(H) \longrightarrow \mathbb{Z}_{3}$ and add to Turán's example all remaining hyperedges $e$ that are not properly colored by $\chi$ (i.e., those $e$ for which $\left.\chi\right|_{e}$ assumes twice some $a \in \mathbb{Z}_{3}$, and once the value $(a-1))$.

As to the upper bound, $\mathcal{H}_{5}=\left\{H_{5,4}, H_{5,5}, H_{5,9}\right\}$, and

$$
\llbracket(e-7 / 9)(1-e) \rrbracket_{1}=\llbracket\left(1-e^{*}\right)\left(2 / 3-e^{*}\right)^{2} \rrbracket_{2} \geq 0,
$$

where $e^{*} \in \mathcal{F}_{3}^{2}$ is a labeled edge.

Since Turán's construction does not contain induced pentagons, we still have $\pi_{\min }\left(I_{4}^{3}, G_{3}, C_{5}\right)=4 / 9$. The situation, however, changes if we forbid not only pentagons but also all of its subgraphs (in the complementary setting of Remark 1, this corresponds to the "hybrid" restriction in which we forbid induced copies of $G_{1}$ and all copies of $\left.G_{4}, C_{5}\right)$. More precisely, let $\widehat{C}_{5} \stackrel{\text { def }}{=}\left\{H_{5,1}, H_{5,6}, H_{5,7}\right\}$.

Theorem 4 (Mubayi, unpublished). $\pi_{\min }\left(I_{4}^{3}, G_{3}, \widehat{C}_{5}\right)=5 / 9$.

Proof. For the lower bound (Mubayi), let $\chi: V(H) \longrightarrow \mathbb{Z}_{3}$ have the same meaning as before, and let $E(H)$ consist of those $e \in[V(H)]^{3}$ for which $\left|e \cap \chi^{-1}(0)\right| \neq 1$.

For the upper bound, let

$$
f_{1} \stackrel{\text { def }}{=} F_{\{1\}}^{N}-2 F_{\{2,3\}}^{N}
$$

and $f_{i}=\gamma^{i-1} f_{1}$, where $\gamma=(1,2,3) \in \Gamma_{N}$ is the cyclic permutation of labeled vertices. Then the quadratic form

$$
Q_{N} \stackrel{\text { def }}{=} 3\left(f_{1}^{2}+f_{2}^{2}+f_{3}^{2}\right)+\left(f_{1}+f_{2}+f_{3}\right)^{2}
$$

is positive semidefinite and

$$
\llbracket(1-e)(e-5 / 9) \rrbracket_{1}=\frac{1}{36} \llbracket Q_{N} \rrbracket_{N}+\frac{1}{10} H_{5,8} \geq 0 .
$$

So far our constraints always involved induced subgraphs. This is not the case in the rest of the paper, so we switch to the dual notation $\pi\left(H_{1}, \ldots, H_{h}\right)$ that is probably more customary to many readers anyway.

Theorem 5 (see $[10]) . \pi\left(H_{5,2}\right)=4 / 9$.

Proof. $\pi\left(H_{5,2}\right) \geq 4 / 9$ is implied by the 3 -graph with a $1 / 3,2 / 3$ partition of the vertices and all edges with exactly two vertices in the larger part [10].

As for the upper bound, in this case $\mathcal{H}_{6}$ consists of 426 different 3 -graphs, and we do not attempt to list all of them. We let (recall that $E$ is the type of size 3 corresponding to an edge)

$$
\begin{aligned}
& f_{1} \stackrel{\text { def }}{=} F_{\{2\}}^{E}+F_{\{3\}}^{E}-F_{\{1,2\}}^{E}-F_{\{1,3\}}^{E} ; \\
& f_{2} \stackrel{\text { def }}{=} 2 F_{\{1\}}^{E}-F_{\{2,3\}}^{E} ; \\
& f_{3} \stackrel{\text { def }}{=} F_{\{1,2,3\}}^{E},
\end{aligned}
$$


and let the quadratic form $Q\left(f_{1}, f_{2}, f_{3}\right)$ be defined by the positive semidefinite matrix

$$
M \stackrel{\text { def }}{=} \frac{1}{24}\left(\begin{array}{ccc}
57 & -26 & -41 \\
-26 & 18 & 6 \\
-41 & 6 & 56
\end{array}\right) .
$$

Then Theorem 5 follows from

$$
\begin{aligned}
\llbracket e(4 / 9-e) \rrbracket_{1} \geq & \frac{8}{9} \llbracket F_{\{2,3\}}^{N}\left(F_{\{1,2\}}^{N}-F_{\{1,3\}}^{N}\right)^{2} \rrbracket_{N}+\frac{4}{3} \llbracket F_{\{2,3\}}^{E}\left(F_{\{1\}}^{E}-\frac{1}{2} F_{\{2,3\}}^{E}\right)^{2} \rrbracket_{E} \\
& +\frac{4}{9} \llbracket F_{\{1\}}^{E}\left(\left(2 F_{\{3\}}^{E}-2 F_{\{2\}}^{E}+F_{\{1,3\}}^{E}-F_{\{1,2\}}^{E}\right)^{2}+Q\left(f_{1}, f_{2}, f_{3}\right)\right) \rrbracket_{E} .
\end{aligned}
$$

6.3. Some other computations. In this section we present, in the style of (1), the results of a few other computations that do not achieve a tight result but still give a significant numerical improvement over previously known bounds.

De Caen [5] proved that $\pi\left(G_{3}\right) \leq 1 / 3$, and this was improved to $1 / 3-10^{-10}$ by Matthias [16]. Mubayi [17] showed that $\pi\left(G_{3}\right) \leq 1 / 3-\left(0.45305 \times 10^{-5}\right)$. Markström and Talbot [15] improved this to $\pi\left(G_{3}\right) \leq 0.3291$.

This time we have 106 admissible graphs on 6 vertices, and the corresponding semidefinite program gives

$$
\pi\left(G_{3}\right) \leq 0.2978 \text {. }
$$

This should be compared with the best known upper bound

$$
\pi\left(G_{3}\right) \geq \frac{2}{7} \approx 0.2857
$$

from [9] that is conjectured to be tight.

One of the motivations for studying $\pi\left(G_{3}\right)$ [17] was the conjecture attributed in [9] to Erdös and Sós that $1 / 4$ is the maximal edge density in a 3 -graph in which all links are bipartite. In these terms, $\pi\left(G_{3}\right)$ corresponds to forbidding triangles in links. If we also forbid cycles of length 5 , that is, the 3 -graph $L_{5} \stackrel{\text { def }}{=}\{(123),(134),(145),(156)$, $(162)\}$, then we can significantly improve (9) to

$$
\pi\left(G_{3}, L_{5}\right) \leq 0.266
$$

(98 admissible 3 -graphs on 6 vertices). Note that in combination with (10), this implies $\pi\left(G_{3}, L_{5}\right)<\pi\left(G_{3}\right)$.

Mubayi and Rödl [19] studied $\pi\left(G_{3}, C_{5}\right)$ and proved that $\pi\left(G_{3}, C_{5}\right) \leq \frac{10}{31} \leq$ 0.3223 . Our analysis on 92 admissible 3 -graphs with 6 vertices shows that

$$
\pi\left(G_{3}, C_{5}\right) \leq 0.2546 .
$$

This also beats $(10)$ and implies $\pi\left(G_{3}, C_{5}\right)<\pi\left(G_{3}\right)$. It is worth noting that since $\pi\left(G_{3}, C_{5}\right)<\pi\left(C_{5}\right)$ also follows from (11) below, the pair $\left\{G_{3}, C_{5}\right\}$ makes yet another example of a nonprincipal pair of 3 -graphs $[1,18]$.

Reference [15] also studied the version $\pi_{2}\left(G_{3}\right)$ when the $G_{3}$-free 3 -graph must also be 2-colorable; that is, its vertex set can be represented as the union of two independent sets. They showed $0.2573 \leq \pi_{2}\left(G_{3}\right)<0.291$. 
For our improvement of their upper bound, we look at the first-order theory (cf. [22, section 2]) of 2-colored, as opposed to 2-colorable graphs. In other words, the models consist of pairs $(H, \chi)$, where $H$ is a $G_{3}$-free 3 -graph and $\chi$ is its (explicit) 2-coloring. There are 1059 admissible models on 6 vertices, and the corresponding semidefinite program gives

$$
\pi_{2}\left(G_{3}\right)<0.2621
$$

The authors of [19] were also interested in $\pi\left(C_{5}\right)$, and they proved that

$$
0.4641<2 \sqrt{3}-3 \leq \pi\left(C_{5}\right) \leq 2-\sqrt{2}<0.586 .
$$

There exist 835 admissible 3 -graphs on 6 vertices, and our calculation shows that

$$
\pi\left(C_{5}\right)<0.4683 \text {. }
$$

This value is pretty close to the lower bound in (11) which brings about an interesting possibility that perhaps the construction from [19] is optimal and, in fact, $\pi\left(C_{5}\right)=$ $2 \sqrt{3}-3$ is an irrational number. While such examples have been recently discovered for 2-colorable 3 -graphs [20], to the best of our knowledge they are still unknown for 3 -graphs without an additional structure.

Acknowledgment. My thanks are due to Dhruv Mubayi for his permission to include here the material from section 6.2. I am also grateful to him, Alexander Kostochka, Oleg Pikhurko, and anonymous referees of this paper for several useful remarks.

\section{REFERENCES}

[1] J. BAlOGH, The Turan density of triple systems is not principal, J. Combin. Theory Ser. A, 100 (1990), pp. 176-180.

[2] J. A. Bondy, Counting subgraphs: A new approach to the Caccetta-Häggkvist conjecture, Discrete Math., 165/166 (1997), pp. 71-80.

[3] B Borchers, CSDP, a C library for semidefinite programming, Optim. Methods Softw., 11 (1999), pp. 613-623.

[4] W. G. Brown, On an open problem of Paul Turán concerning 3-graphs, in Studies in Pure Mathematics, Birkhäuser, Boston, 1983, pp. 91-93.

[5] D. DE CAEn, Extension of a theorem of Moon and Moser on complete subgraphs, Ars Combin., 16 (1983), pp. 5-10.

[6] D. DE CAEn, The current status of Turán problem on hypergraphs, in Extremal Problems for Finite Sets, Vol. 3, Bolyai Society Mathematical Studies, Visegrád, Hungary, 1991, pp. $187-197$.

[7] F. Chung And L. Lu, An upper bound for the Turán number $t_{3}(n, 4)$, J. Combin. Theory (A), 87 (1999), pp. 381-389.

[8] D. G. Fon-DER-FlaAss, Method for construction of $(3,4)$-graphs, Mathematical Notes, 44 (1988), pp. 781-783; Matematicheskie Zametki, 44 (1988), pp. 546-550 (in Russian).

[9] P. Frankl and Z. Füredi, An exact result for 3-graphs, Discrete Math., 50 (1984), pp. 323328.

[10] Z. Füredi, O. Pikhurko, and M. Simonovits, The Turán density of the hypergraph $\{a b c$, ade, bde.cde\}, Electron. J. Combin., R18 (2003).

[11] J. HladkÝ, D. KRÁL', And S. Norine, Counting flags in triangle-free digraphs, manuscript, available at http://arxiv.org/abs/0908.2791, 2009.

[12] A. V. Kostochka, A class of constructions for Turán's (3,4)-problem, Combinatorica, 2 (1982), pp. 187-192.

[13] L. LovÁsz, Graph homomorphisms: Open problems, manuscript, available at http://www.cs. elte.hu/ lovasz/problems.pdf, 2008.

[14] W. Mantel, Problem 28, solution by H. Gouwentak, W. Mantel, J. Teixeira de Mattes, F. Schuh, and W.A. Wythoff, Wiskundige Opgaven, 10 (1907), pp. 60-61. 
[15] K. Markström and J. TAlbot, On the density of 2-colorable 3-graphs in which any four points span at most two edges, J. Comb. Des., 18 (2010), pp. 105-114.

[16] U. Matthias, Hypergraphen ohne vollständige r-partite Teilgraphen, Ph.D. thesis, Heidelberg, 1994.

[17] D. Mubayi, On hypergraphs with every four points spanning at most two triples, Electron. J. Combin., N10 (2003).

[18] D. Mubayi And O. Pikhurko, Constructions of nonprincipal families in extremal hypergraph theory, Discrete Math., 308 (2008), pp. 4430-4434.

[19] D. Mubayi And V. RöDl, On the Turán number of triple systems, J. Combin. Theory Ser. A, 100 (2002), pp. 136-152.

[20] D. Mubayi and J. Talbot, Extremal problems for t-partite and t-colorable hypergraphs, Electron. J. Combin., 15 (2008). Research paper 26.

[21] O. Piknurko, The minimum size of 3-graphs without a 4-set spanning no or exactly three edges, manuscript, 2009.

[22] A. Razborov, Flag algebras, J. Symbolic Logic, 72 (2007), pp. 1239-1282.

[23] A. RazBorov, On the minimal density of triangles in graphs, Combin. Probab. Comput., 17 (2008), pp. 603-618.

[24] A. F. Sidorenko, What we know and what we do not know about Turán numbers, Graphs Combin., 11 (1995), pp. 179-199.

[25] P. Turán, Egy gráfelméleti szélsöértékfeladatról, Mat. és Fiz. Lapok, 48 (1941), pp. 436-453. 